\title{
Dynamics and Thermodynamics of Linear Quantum Open Systems
}

\author{
Esteban A. Martinez ${ }^{1, *}$ and Juan Pablo Paz ${ }^{1,2}$ \\ ${ }^{1}$ Departamento de Física, FCEyN, UBA, Ciudad Universitaria Pabellón 1, 1428 Buenos Aires, Argentina \\ ${ }^{2}$ IFIBA CONICET, UBA, FCEyN, UBA, Ciudad Universitaria Pabellón 1, 1428 Buenos Aires, Argentina \\ (Received 16 July 2012; revised manuscript received 26 September 2012; published 26 March 2013)
}

\begin{abstract}
We analyze the evolution of the quantum state of networks of quantum oscillators coupled with arbitrary external environments. We show that the reduced density matrix of the network always obeys a local master equation with a simple analytical solution. We use this to study the emergence of thermodynamical laws in the long time regime demonstrating two main results: First, we show that it is impossible to build a quantum absorption refrigerator using linear networks (thus, nonlinearity is an essential resource for such refrigerators recently studied by Levy and Kosloff [Phys. Rev. Lett. 108, 070604 (2012)] and Levy et al. [Phys. Rev. B 85, 061126 (2012)]). Then, we show that the third law imposes constraints on the low frequency behavior of the environmental spectral densities.
\end{abstract}

Deriving the laws of thermodynamics from a quantum substrate is relevant not only for fundamental but also for practical reasons [1-3]. In the macroscopic domain such laws determine the ultimate limits on cooling and work extraction. However, when quantum effects dominate, thermodynamic laws must be derived (not assumed), which is still a controversial issue. In particular, the ultimate limitations on cooling, imposed by the third law have been recently debated $[2,4]$. Moreover, a variety of quantum devices have been proposed to act as engines or refrigerators [2,5]. Among them, the quantum absorption refrigerator [2], whose description does not admit a phenomenological approach. The resources required for quantum refrigerators to operate are not fully known. Our work is a step towards determining some of the essential resources required to build such machines.

We study the dynamics and the thermodynamics of arbitrary networks of $N$ oscillators moving in $D$ dimensions while coupled with external reservoirs as shown in Fig. 1. This is a generalization of the quantum Brownian motion (QBM) model [6-9]. Being the paradigm for an open quantum system, this model has been used to study the emergence of classicality through decoherence [9]. Our work not only has applications to quantum refrigerators but also to other systems where a detailed understanding of heat transport [10] and decoherence is required. This is the case for trapped-ion quantum simulators [11,12]. It also may help in understanding other natural processes such as the high efficiency of energy transfer in light harvesting complexes [13].

We present four main results. First, we show that the dynamics is such that (i) the quantum state always satisfies a local master equation, and (ii) such equation always has a simple analytical solution. Then we use these results to study the long time limit. We present a simple derivation of thermodynamical laws and prove two new results: (iii) We show that it is impossible to create a quantum absorption refrigerator using linear networks. Such refrigerators have no movable parts and induce the energy to flow away from a cold reservoir by coupling it with a system that is itself coupled with other (hot) reservoirs. In the quantum regime they were proposed by Kosloff and others [2] using a nonlinear model. Our work implies that, as nonlinearity is an essential resource, a quantum absorption refrigerator could never be built using harmonic systems such as trapped ions [11] or nanomechanical systems that are ideal to implement other quantum machines [14]. Finally, we show that (iv) the third law (as stated by Nernst: entropy flow vanishes at zero temperature) imposes a constraint on the environmental spectral density, which for low frequencies must behave as $\omega^{\nu}$ with $\nu>0$.

We consider the total Hamiltonian $H_{T}=H_{S}+H_{E}+$ $H_{\text {int }}$, where the system Hamiltonian is $H_{S}=P^{T} P / 2+$ $X^{T} V X / 2(X$ and $P$ are column vectors storing the $K=$ $N D$ system's coordinates and momenta). The $K \times K$ matrix $V$ defines the network's couplings (we consider unit masses and use superscript $T$ for the transpose). The environmental Hamiltonian is $H_{E}=\sum_{\alpha} H_{\alpha}$ with $H_{\alpha}=$ $\sum_{k}\left(\pi_{k}^{(\alpha)^{2}} / 2 m_{k}+m_{k} \omega_{k}^{2} q_{k}^{(\alpha)^{2}} / 2\right)$. The interaction is $H_{\text {int }}=$ $\sum_{\alpha, i, k} C_{i k}^{(\alpha)} x_{i} q_{k}^{(\alpha)}$. We study the evolution of the reduced density matrix of the system $\rho=\operatorname{Tr}_{E}\left(\rho_{S E}\right)$.

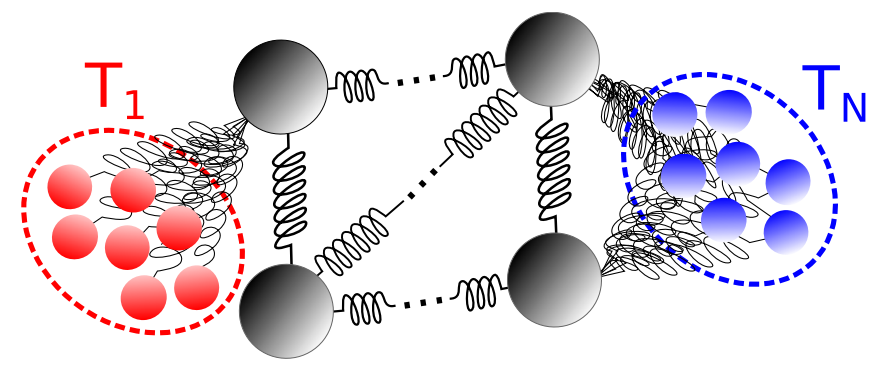

FIG. 1 (color online). We analyze the most general network of interacting oscillators coupled with bosonic reservoirs characterized by arbitrary spectral densities and initial thermal states. 
The following two results are valid if the time evolution preserves Gaussian states (i.e., if the total Hamiltonian is quadratic in $P$ and $X$ ). In this case (i) $\rho(t)$ satisfies the timelocal master equation:

$$
\begin{aligned}
\dot{\rho}= & -i\left[H_{R}(t), \rho\right]-i \Gamma_{i j}(t)\left[x_{i},\left\{p_{j}, \rho\right\}\right] \\
& -i \tilde{\Gamma}_{i j}(t)\left[p_{i},\left\{x_{j}, \rho\right\}\right]-D_{i j}(t)\left[x_{i},\left[x_{j}, \rho\right]\right] \\
& -\tilde{D}_{i j}(t)\left[p_{i},\left[p_{j}, \rho\right]\right]-F_{i j}(t)\left[x_{i},\left[p_{j}, \rho\right]\right] .
\end{aligned}
$$

Here, $H_{R}(t)=P^{T} M_{R}^{-1}(t) P / 2+X^{T} V_{R}(t) X / 2+f^{T}(t) X+$ $\tilde{f}^{T}(t) P$ is a renormalized Hamiltonian with time dependent couplings, masses and forces $V_{R}(t), M_{R}(t), f(t)$, and $\tilde{f}(t)$. The master equation includes nonunitary effects as relaxation [through $\Gamma(t)$ and $\tilde{\Gamma}(t)$ ] and diffusion [through $D(t)$, $\tilde{D}(t)$ and $F(t)]$. The second result is (ii) the state $\rho(t)$ can be written as a function of time in a simple way using the characteristic function defined as $\chi(\kappa, t)=\operatorname{Tr}[\rho(t) \hat{D}(\kappa)]$ (where the displacement operator is $\hat{D}(\kappa)=\exp \left[-i\left(P k_{p}-\right.\right.$ $\left.X k_{x}\right)$ ], where $\kappa=\left(k_{x}, k_{p}\right)$ is a $2 K$-component vector defining momentum and position displacements). This function provides a complete description of the state and satisfies that

$\chi(\kappa, t)=\chi(\Phi(t) \kappa, 0) \exp \left[-\frac{1}{2} \kappa^{T} \Sigma(t) \kappa\right] \exp \left[i \Pi^{T}(t) \kappa\right]$.

The $2 K \times 2 K$ matrices $\Phi(t)$ and $\Sigma(t)$ and the $2 K$-vector $\Pi(t)$ depend on time. Results (1) and (2) imply that for arbitrary Gaussian channels the state evolves through a combination of a phase space flow and a Gaussian modulation (the environment only induces renormalization, friction, and diffusion).

We sketch here the derivation of Eqs. (1) and (2). To the best of our knowledge they are both new results (details can be found in the Supplemental Material [1]). Equation (1) generalizes the master equation obtained for QBM in Ref. [6]. Equation (2) generalizes a classical result by Rieder, Lebowitz, and Lieb (see Ref. [10] for references). For QBM Eq. (2) was first discussed in Ref. [8]. Both results follow from properties of the evolution super-operator for $\rho(t)$, which is such that $\rho(t)=\mathcal{J}[\rho(0)]$. Any superoperator preserving Gaussian quantum states (together with Hermiticity and trace) can be written in the position representation as $\mathcal{J}\left(z, z^{\prime}, t ; z_{0}, z_{0}^{\prime}, 0\right) \equiv\left\langle z\left|\mathcal{J}\left(\left|z_{0}^{\prime}\right\rangle\left\langle z_{0}\right|\right)\right| z^{\prime}\right\rangle=$ $\operatorname{det}\left(b_{3}\right) \times e^{i\left(\xi^{T} b_{1} Z+\xi^{T} b_{2} Z_{0}+\xi_{0}^{T} b_{3} Z+\xi_{0}^{T} b_{4} Z_{0}\right)} e^{i\left(c_{1}^{T} \xi+c_{2}^{T} \xi_{0}\right)} \times$ $e^{-\xi^{T} a_{1} \xi-\xi^{T} a_{2} \xi_{0}-\xi_{0}^{T} a_{3} \xi_{0}} /(2 \pi)^{K}$ [here $\xi=z-z^{\prime}$ and $Z=$ $\left.\left(z+z^{\prime}\right) / 2\right]$. The matrices $b_{m}$ and $a_{m}$ together with the vectors $c_{m}$ parametrize any quantum evolution and depend on the microscopic model, as described below. Equation (1) is obtained following [7,9]: Computing the time derivative of the propagator we can show that $\dot{\mathcal{J}}\left(z, z^{\prime}, t ; z_{0}, z_{0}^{\prime}, 0\right)=P\left(Z, \xi, Z_{0}, \xi_{0}\right) \times \mathcal{J}\left(z, z^{\prime}, t ; z_{0}, z_{0}^{\prime}, 0\right)$, where $P$ is a quadratic polynomial of its arguments. All terms in $P \times \mathcal{J}$ that are proportional to $Z_{0}$ and $\xi_{0}$ can be rewritten in a simple way. Thus, using the Gaussian nature of $\mathcal{J}$ we can show that they can be expressed as a linear combination of terms proportional to $Z, \xi$ and to the derivatives of $\mathcal{J}$ with respect to them. Doing this, the master equation (1) is obtained. Equation (2) directly follows by integrating over initial coordinates using the Gaussian propagator [1].

Matrices in (1) and (2) are determined by those appearing in the propagator $\mathcal{J}$. For the generalized QBM model defined by the Hamiltonian $H_{T}$ we can compute $\mathcal{J}$ using path integral techniques [15]. As described in Ref. [1] $\mathcal{J}$ depends only on two properties of the environment: the initial temperatures $T_{\alpha}$ and the spectral density, defined as $I(\omega)=\sum_{\alpha} I^{(\alpha)}(\omega)$, where $I_{i j}^{(\alpha)}(\omega)=\sum_{k} C_{i k}^{(\alpha)} C_{j k}^{(\alpha)} \delta(\omega-$ $\left.\omega_{k}\right) / 2 m_{k} \omega_{k}$ (these are real, symmetric and positive $K \times K$ matrices). These characters appear in two kernels: The dissipation kernel defined as $\gamma(\tau)=\int_{0}^{\infty} d \omega I(\omega) \times$ $\cos (\omega \tau) / \omega$ and the noise kernel $\nu(\tau)=\int_{0}^{\infty} d \omega \hat{\nu}(\omega) \times$ $\cos (\omega \tau)$ [where $\hat{\nu}(\omega)=\sum_{\alpha} I^{(\alpha)}(\omega) \operatorname{coth}\left(\omega / 2 k_{B} T_{\alpha}\right)$ ]. As shown in Ref. [1], all coefficients in (1) and (2) depend on the spectral density throughout the $K \times K$ matrix $G(t)$, which is the unique solution of

$$
\ddot{G}+V_{R} G+2 \int_{0}^{t} d \tau \gamma(t-\tau) \dot{G}(\tau)=0,
$$

with initial conditions $G(0)=0$ and $\dot{G}(0)=\hat{1}$ (the renormalized potential $V_{R}=V-2 \gamma(0)$ is the asymptotic limit of $V_{R}(t)$ for large values of the cutoff). Equation (3) can be solved using the Laplace transform [the transformed of $G(t)$ is denoted as $\hat{G}(s)]$. Thus, we find $\hat{G}(s)=\left[s^{2} I+V_{R}+\right.$ $2 s \hat{\gamma}(s)]^{-1}$, where $\hat{\gamma}(s)=\int_{0}^{\infty} d \omega I(\omega) s^{2} /\left(\omega^{2}+s^{2}\right)$ is the transform of the dissipation kernel. All coefficients in (1) are listed in the Supplemental Material [1]. For example, in this case we find that there is no mass renormalization, no renormalized forces, no momentum diffusion and only normal dissipation [i.e., $M(t)=\hat{1}, f(t)=0=\tilde{f}(t), \tilde{\Gamma}(t)=$ $\tilde{D}(t)=0]$. Moreover, the dissipation matrix is $2 \Gamma(t)=$ $\left(\ddot{\ddot{G}} \dot{G}^{-1} G-\ddot{G}\right)\left(\dot{G}-\ddot{G} \dot{G}^{-1} G\right)^{-1}$. In turn, for the generalized QBM model, the solution (2) is such that $\Pi(t)=0$ and the matrices $\Phi(t)$ and $\Sigma(t)$ are

$$
\begin{aligned}
& \Phi(t)=\left[\begin{array}{ll}
\dot{G}(t) & G(t) \\
\ddot{G}(t) & \dot{G}(t)
\end{array}\right] \text { and } \\
& \Sigma(t)=\left[\begin{array}{ll}
\sigma^{(0,0)}(t) & \sigma^{(0,1)}(t) \\
\sigma^{(1,0)}(t) & \sigma^{(1,1)}(t)
\end{array}\right] .
\end{aligned}
$$

Here, the $K \times K$ submatrices of $\Sigma(t)$ are $\sigma^{(n, m)}(t)=$ $\int_{0}^{t} \int_{0}^{t} d t_{1} d t_{2} G^{(n)}\left(t_{1}\right) \nu\left(t_{1}-t_{2}\right) G^{(m)}\left(t_{2}\right)$. Diffusion matrices in (1) are also determined by $\Sigma(t)$. For example, $D(t)=\operatorname{Sym}\left[V_{R}(t) \sigma^{(0,1)}(t)+2 \Gamma(t) \sigma^{(1,1)}(t)+\dot{\sigma}^{(1,1)}(t)\right]$.

As seen from (2), the final state is independent of the initial one if and only if $G(t)$ decays (indeed, this is the case because $\chi(0, t)=1)$. Assuming that this condition is satisfied we can derive important properties of the stationary regime $[G(t)$ decays for certain spectral densities and 
coupling matrices but it exhibits revivals for finite reservoirs]. To study energy transfer between the system and the environments we compute the time derivative of the expectation value of the renormalized Hamiltonian; i.e., $d\left(\left\langle H_{R}\right\rangle\right) / d t$. The master equation implies that $d\left(\left\langle H_{R}\right\rangle\right) / d t=\operatorname{tr}\left(D-2 \Gamma \sigma^{(1,1)}\right.$ ) (where the trace involves a summation over all sites of the network). Replacing the formula for the diffusion matrix [1] we get that, in the stationary regime, $d\left(\left\langle H_{R}\right\rangle\right) / d t=\operatorname{tr}\left(V_{R} \sigma^{(0,1)}\right)$. We will consider first the case where the network is divided into nonoverlapping regions $S_{\alpha}(\alpha=1, \ldots, R)$. We will also assume that each region is coupled with an environment $E_{\alpha}$ (these assumptions will be relaxed below). In this case we can write $d\left(\left\langle H_{R}\right\rangle\right) / d t=\sum_{\alpha} \dot{Q}_{\alpha}$. Here, $\dot{Q}_{\alpha}$ is the heat current entering $S_{\alpha}$ and is given as $\dot{Q}_{\alpha}=\operatorname{tr}\left(P_{S_{\alpha}} V_{R} \sigma^{(0,1)}\right)$ (where $P_{S_{\alpha}}$ is the projector onto $S_{\alpha}$ ). In fact, $\dot{Q}_{\alpha}$ is equal to the mean value of the power transmitted by $E_{\alpha}$ to the system. In the stationary limit we obtain that the conservation law $\sum_{\alpha} \dot{Q}_{\alpha}=0$ is satisfied. Also, we obtain explicit expressions for the heat currents using that $\sigma^{(n, m)} \rightarrow$ $\operatorname{Re} \int_{0}^{\infty} d \omega \omega^{n+m} i^{m-n} \hat{G}(i \omega) \hat{\nu}(\omega) \hat{G}(-i \omega)$. Then,

$$
\dot{Q}_{\alpha}=\sum_{\beta} \int_{0}^{\infty} \omega d \omega \dot{Q}_{\alpha \beta}(\omega) \operatorname{coth}\left(\omega / 2 k_{B} T_{\beta}\right),
$$

where the heat transfer matrix $\dot{Q}$ is such that

$$
\begin{aligned}
\dot{\mathcal{Q}}_{\alpha \beta}(\omega) & =\operatorname{Im} \operatorname{tr}\left[P_{S_{\alpha}} V_{R} \hat{G}(i \omega) I^{(\beta)}(\omega) \hat{G}(-i \omega)\right] \\
& =-\pi \operatorname{tr}\left[I_{\alpha}(\omega) \hat{G}(i \omega) I_{\beta}(\omega) \hat{G}^{\dagger}(\omega)\right] \leq 0 \quad \alpha \neq \beta .
\end{aligned}
$$

The inequality in the second line of (6) follows from the positivity of the spectral densities $I_{\alpha}$ and $I_{\beta}$. As shown in Ref. [1], to obtain (6) we used the definition of $\hat{G}(s)$ together with the condition $\operatorname{Re}[2 \omega \hat{\gamma}(i \omega)]=\pi I(\omega)$ (which is nothing but the simplest form of the fluctuation dissipation theorem [6]). In what follows, we will only need to use the following general properties of the heat transfer matrix $\dot{Q}_{\alpha \beta}(\omega)$ that are a direct consequence of (6): (i) $\dot{\mathcal{Q}}_{\alpha \beta}(\omega)=\dot{\mathcal{Q}}_{\beta \alpha}(\omega)$, (ii) $\dot{\mathcal{Q}}_{\alpha \beta}(\omega) \leq 0$ if $\alpha \neq \beta$, and (iii) $\sum_{\alpha} \dot{\mathcal{Q}}_{\alpha \beta}(\omega)=0$.

Using the above results we can derive the following thermodynamic laws: (a) Equilibrium: Energy flows through the system if and only if there is a temperature gradient. This is due to the fact that the heat flow into the region $S_{\alpha}$ is

$$
\dot{Q}_{\alpha}=-2 \sum_{\beta \neq \alpha} \int_{0}^{\infty} \omega d \omega \dot{\mathcal{Q}}_{\alpha \beta}(\omega)\left[n_{\alpha}(\omega)-n_{\beta}(\omega)\right],
$$

where $n_{\alpha}(\omega)=\left[\operatorname{coth}\left(\omega / 2 k_{B} T_{\alpha}\right)-1\right] / 2$. As $\dot{Q}_{\alpha \beta}(\omega) \leq 0$, the heat current $\dot{Q}_{\alpha}$ vanishes if and only if $T_{\alpha}=T_{\beta}$ for all $\alpha, \beta$. This is equivalent to the 0th law of thermodynamics: When all the environments have the same temperature then energy does not flow through the system and equilibrium is reached. (b) Heat flows from the hot to the cold reservoir. This is the Clausius version of the second law of thermodynamics and can be shown as follows: If $T_{\alpha}$ is the highest temperature, then $n_{\alpha}(\omega)>n_{\beta}(\omega)$ for all $\beta$. Therefore, as $\dot{Q}_{\alpha \beta}(\omega) \leq 0$ Eq. (7) implies that $\dot{Q}_{\alpha} \geq 0$. Thus, the hottest reservoir always injects energy in the system and this energy is absorbed by the other reservoirs (as implied by the conservation law). Another formulation of the second law in terms of the entropy flow $\dot{S}=\sum_{\alpha} \dot{Q}_{\alpha} / T_{\alpha}$ states that $\dot{S} \leq 0$, which follows from (7), since $\dot{S}=-\int_{0}^{\infty} d \omega \sum_{\alpha \neq \beta} \dot{Q}_{\alpha \beta}(\omega)\left(1 / T_{\beta}-1 / T_{\alpha}\right) \times$ $\left(n_{\beta}(\omega)-n_{\alpha}(\omega)\right) \leq 0$.

The above argument can be used to show that the coldest reservoir always absorbs energy from the system. In fact, if $T_{\alpha}<T_{\beta}$ for all $\beta$ Eq. (7) implies that $\dot{Q}_{\alpha} \leq 0$. This implies that harmonic networks cannot be used to build a quantum absorption refrigerator. Such quantum machines (that have no movable pieces) were discussed by Kosloff and co-workers [2]. Our result implies that nonlinearity is an essential resource for absorption refrigerators. As stated, the no-go theorem for linear absorption refrigerators is valid for any spectral density.

The assumptions we made in the above derivation can be relaxed. Thus, we assumed that each region of the system couples with a single environment. We can generalize this as follows: We assume that the region $\alpha$ couples with $N_{\alpha}$ environments labeled by the index $a_{\alpha}$ (each having temperature $T_{a_{\alpha}}$ ). For simplicity, we also assume that all the spectral densities $I^{\left(\alpha, a_{\alpha}\right)}$ are the same (this can also be relaxed), i.e., $\sum_{a_{\alpha}} I^{\left(\alpha, a_{\alpha}\right)}(\omega)=N_{\alpha} I^{(\alpha)}(\omega)$. In this case we can show that the heat flow into the region $S_{\alpha}$ is given by Eq. (7), where the temperature dependent factor $\left[n_{\alpha}(\omega)-\right.$ $\left.n_{\beta}(\omega)\right]$ must be replaced by a factor $\left[\tilde{n}_{\alpha}(\omega)-\tilde{n}_{\beta}(\omega)\right]$, where $\tilde{n}_{\alpha}(\omega)=\sum_{a_{\alpha}} n_{a_{\alpha}}(\omega) / N_{\alpha}$ is the average number of excitations of the environments coupled with $S_{\alpha}$. In the high temperature limit, the temperature dependent factor in (7) becomes proportional to the difference between the average temperature of the reservoirs, but this factor depends nonlinearly on the temperatures otherwise. Thus, the second law in this case states that the heat always flows into the region that is coupled with the reservoirs with the largest average number of excitations (or the largest average temperature in the high temperature limit). Equivalently, heat always flows away from the region coupled with the reservoirs with the smallest average number of excitations (temperature). Of course, the environment with the lowest temperature may be coupled with a region which is itself coupled with hotter environments in such a way that the average number of excitations is not the lowest one. Then, heat would flow away from such environments. However, it is clear that by enlarging the definition of "the system" and by using the previous argument, one can prove that the environment with the lowest temperature would absorb heat.

The other assumption in the above derivation is that different regions $S_{\alpha}$ do not overlap. This can also be generalized using the fact that the obtained results are valid for arbitrary networks. Thus, the case of overlapping 
regions can be analyzed as follows: Consider a network with regions $S_{2} \subset S_{1}$. If $S_{1,2}$ couple with different environments $E_{1,2}$, this situation is physically equivalent to that of a network where $S_{1}$ couples with $E_{1}$ while $S_{2}$ couples strongly with another network $S_{2}^{\prime}$, which is itself coupled with $E_{2}$. In such a case, all previous results directly apply. Therefore, the no-go theorem for quantum absorption refrigerators applies to all linear networks.

The above results are valid for arbitrary spectral densities. However, we now show that the validity of the third law imposes constraints on $I(\omega)$. To see this, we consider environments coupled with single sites, with spectral densities $I_{j j}^{(j)}(\omega)=\gamma_{j} \omega^{p_{j}} \theta(\omega)(\theta(\omega)$ is a cutoff function vanishing when $\omega \geq \Lambda$ and $\gamma_{j}$ a coupling constant). When $p_{j}=1$ the environment is Ohmic while it is super-Ohmic (sub-Ohmic) for $p_{j}>1\left(p_{j}<1\right)$. Using this, we now prove that (c) The third law emerges if $p_{j}>0$. Thus, the heat flow into the $j$ th site is

$$
\begin{aligned}
\dot{Q}_{j}= & 2 \pi \sum_{k \neq j} \int_{0}^{\infty} \omega^{1+p_{j}+p_{k}} \gamma_{j} \gamma_{k} \theta^{2}(\omega) d \omega\left|\hat{G}(i \omega)_{j k}\right|^{2} \\
& \times \sum_{a \geq 1}\left(e^{-a \omega / k_{B} T_{j}}-e^{-a \omega / k_{B} T_{k}}\right) .
\end{aligned}
$$

For low temperatures the integral is dominated by low frequencies. Taylor expanding the integrand and assuming that all $T_{j}$ are close to the average $\bar{T}$ we find

$\dot{Q}_{j}=\sum_{k \neq j} \gamma_{j} \gamma_{k} \bar{T}^{1+p_{j}+p_{k}}\left(T_{j}-T_{k}\right)\left(2+p_{j}+p_{k}\right) \alpha_{k j}$,

where $\quad \alpha_{l j}=2 \pi\left(k_{B}\right)^{2+p_{j}+p_{k}}\left|\hat{G}(0)_{j k}\right|^{2} \Gamma\left(2+p_{j}+p_{k}\right) \zeta(2+$ $\left.p_{j}+p_{k}\right)$. Thus, for two reservoirs with the same spectral density (i.e., $p_{A}=p_{B}=p$ ) and a small temperature difference we find that the heat flow is $\dot{Q}_{A}=2(p+$ 1) $\gamma^{2} \alpha_{A B} \bar{T}^{2 p+1} \Delta T$. This implies that the entropy flow into site- $A$ is $\dot{S}_{A}=\dot{Q}_{A} / T_{A} \propto \bar{T}^{2 p}$. Thus, if $p>0$ the entropy flow vanishes when $\bar{T} \rightarrow 0$, which is nothing but Nernst's (static) version of the third law.

The results reported here concern the evolution of open networks of quantum oscillators. In this case (i) we deduced an exact master equation and (ii) we obtained its explicit solution. We used this to show how thermodynamic laws emerge from first principles [they emerge for arbitrary environments whenever $G(t)$ decays for long times]. We proved (iii) a no-go theorem for linear absorption refrigerators identifying nonlinearity as an essential resource for such machines (ruling out possible implementations using harmonic systems such as trapped ions, phonons, or nanomechanical oscillators [14]). Also, we showed (iv) the validity of the third law imposes a constraint on the low frequency behavior of the spectral densities. Very few derivations of the third law are available. Our treatment enabled us to obtain a straightforward derivation of the "static" version of such law (as postulated by Nernst: the entropy flow from any substance is zero at absolute zero).
The validity of the dynamical version of the third law (the unattainability of zero temperature in finite time) seems to impose even stronger constraints on the spectral density $I(\omega)$, as recently reported $[2,4]$ : the static version of the third law is consistent with sub-Ohmic, Ohmic, and superOhmic environments whereas the sub-Ohmic ones are excluded according to Ref. [2]. Finally, we point out that it is possible to extend our results to driven systems with quadratic Hamiltonians. This could be done using the above methods, computing time averaged quantities with an approach similar to that of Refs. [2,16].

This work was supported by ANPCyT, CONICET, and Ubacyt.

*Present address: Institute fur Experimentalphysik., Universität Innsbruck, Technikerstraße 25, A-6020 Innsbruck, Austria.

[1] See Supplemental Material at http://link.aps.org/ supplemental/10.1103/PhysRevLett.110.130406 for a detailed derivation of all the equations.

[2] A. Levy and R. Kosloff, Phys. Rev. Lett. 108, 070604 (2012); A. Levy, R. Alicki, and R. Kosloff, Phys. Rev. B 85, 061126 (2012).

[3] M. Michel, G. Mahler, and J. Gemmer, Phys. Rev. Lett. 95, 180602 (2005); K. Saito, Europhys. Lett. 61, 34 (2003); I. Kim and G. Mahler, Phys. Rev. E 81, 1 (2010); J. Gemmer, M. Michel, and G. Mahler, Quantum Thermodynamics (Springer, New York, 2010).

[4] M. Kolar, D. Gelbwaser-Klimovsky, R. Alicki, and G. Kurizki, Phys. Rev. Lett. 109, 090601 (2012).

[5] R. Kosloff, J. Chem. Phys. 80, 1625 (1984); S. Lloyd, Phys. Rev. A 56, 3374 (1997); R. Kosloff, E. Geva, and J. M. Gordon, Appl. Phys. 87, 8093 (2000).

[6] B. L. Hu, J. P. Paz, and Y. Zhang, Phys. Rev. D 45, 2843 (1992), and references therein.

[7] L. Dávila Romero and J. P. Paz, Phys. Rev. A 55, 4070 (1997).

[8] C.H. Fleming, B. L. Hu, and A. Roura, Ann. Phys. (Amsterdam) 326, 1207 (2011).

[9] J.P. Paz and W.H. Zurek, Fundamentals of Quantum Information (Springer, New York, 2002), p. 77.

[10] B. Dhar, Adv. Phys. 57, 457 (2008).

[11] T. Pruttivarasin, M. Ramm, I. Talukdar, A. Krouter, and H. Haffner, New J. Phys. 13, 075012 (2011).

[12] G. D. Lin and L. M. Duan, New J. Phys. 13, 075015 (2011).

[13] M. Mohseni, P. Rebentrost, S. Lloyd, and A. AspriuGuzik, J. Chem. Phys. 129, 174106 (2008); M. B. Plenio and S. F. Huelga, New J. Phys. 10, 113019 (2008).

[14] O. Abah, J. Roßnagel, G. Jacob, S. Deffner, F. SchmidtKaler, K. Singer, and E. Lutz, Phys. Rev. Lett. 109, 203006 (2012); C. Chamon, E. R. Mucciolo, I. Arrachea, and R. B. Capaz, Phys. Rev. Lett. 106, 135504 (2011).

[15] R. P. Feynman and F. L. Vernon, Ann. Phys. (N.Y.) 24, 118 (1963); see also H. Grabert, P. Schramm, and G. L. Ingold, Phys. Rep. 168, 115 (1988); A. O. Caldeira and A. J. Leggett, Physica (Amsterdam) 121, 587 (1983).

[16] L. Arrachea, M. Moskalets, and L. Martin-Moreno, Phys. Rev. B 75, 245420 (2007). 